\title{
Mars Tumbleweed Simulation Using Singular Perturbation Theory
}

\author{
Behzad Raiszadeh, \\ NASA Langley Research Center, Hampton, Va., 23681 \\ Phillip Calhoun \\ NASA Goddard Space Flight Center, Greenbelt, Md.,20771
}

The Mars Tumbleweed is a new surface rover concept that utilizes Martian winds as the primary source of mobility. Several designs have been proposed for the Mars Tumbleweed, all using aerodynamic drag to generate force for traveling about the surface. The Mars Tumbleweed, in its deployed configuration, must be large and lightweight to provide the ratio of drag force to rolling resistance necessary to initiate motion from the Martian surface. This paper discusses the dynamic simulation details of a candidate Tumbleweed design. The dynamic simulation model must properly evaluate and characterize the motion of the tumbleweed rover to support proper selection of system design parameters. Several factors, such as model flexibility, simulation run times, and model accuracy needed to be considered in modeling assumptions. The simulation was required to address the flexibility of the rover and its interaction with the ground, and properly evaluate its mobility. Proper assumptions needed to be made such that the simulated dynamic motion is accurate and realistic while not overly burdened by long simulation run times. This paper also shows results that provided reasonable correlation between the simulation and a drop/roll test of a tumbleweed prototype.

\section{Nomenclature}

$F_{C} \quad$ Compressive force due to damping

$F_{K} \quad$ Compressive force due to stiffness

$F_{T} \quad$ Net compressive force

$F_{N} \quad$ Normal ground reaction force

$F_{F} \quad$ Friction force

$F_{W}$ Constraint force

$L \quad$ Instantaneous length of each leg

$L_{0} \quad$ Uncompressed length of each leg

$\dot{L} \quad$ Rate of change of the leg length

$\vec{M} \quad$ vector from the surface reference point to the leg contact with the ground

$\hat{n} \quad$ Unit vector perpendicular to the ground 
$\vec{O}$ Position vector of the tumbleweed center of mass in the inertial ground frame

$\dot{\vec{O}} \quad$ Velocity vector of the tumbleweed center of mass in the inertial ground frame

$\vec{P} \quad$ Position vector of the surface reference point in the inertial ground frame

$\hat{u} \quad$ Radial leg direction unit vector in the inertial ground frame

$\dot{\hat{u}} \quad$ Rate of change of the unit vector along the leg direction in the inertial ground frame

$\hat{v} \quad$ Unit vector in direction of the Tumbleweed leg tip velocity vector projected onto the ground plane

$\hat{w} \quad$ Constraint force unit vector

$\mu$ ground friction coefficient

$V \quad$ Magnitude of the net velocity of the tip

$V_{F} \quad$ Velocity of the tip projected onto the ground plane

\section{Introduction}

The NASA Langley Research Center has been developing a novel concept for a Mars planetary rover called the Mars Tumbleweed. ${ }^{1}$ This rover concept utilizes in-situ Martian winds to traverse the Mars surface. Deriving mobility from the winds enables this vehicle to cover vast regions of the Mars terrain not possible with current Mars rover technologies. The long-range mobility and targeting of specific sites could be achieved with some degree of control. This capability may enhance or even enable surface missions for surveying and scouting large areas for locating sites for further robotic study or human exploration. ${ }^{1}$ These considerations have motivated the design of a novel Tumbleweed concept, called the Eggbeater-Dandelion. ${ }^{2}$ This paper describes a dynamic model of this concept developed to study the system characteristics required for maneuvering along selected Mars terrain.

\section{Eggbeater-Dandelion Tumbleweed Concept Design}

The Eggbeater-Dandelion is one of many concepts considered for the design of the Tumbleweed rover. Several factors were considered in the trade between different concepts. ${ }^{1}$ These factors include structural considerations, packaging, drag, load path to the core, ability to absorb impact during rolling and bouncing on the surface and weight. The Eggbeater-Dandelion was envisioned as a suitable design that could demonstrate superior performance in all design considerations. Figure 1 shows the Eggbeater-Dandelion design geometry with the core as a truncated icosahedron (12 vertices), which resembles a soccer ball. Each leg resembles an eggbeater or whisk, hence is given the name "Eggbeater leg" or simply "Eggbeater". The structural configuration of the Eggbeaters has a softening-type radial stiffness characteristic. For details of the design see Reference 2. 


\section{Figure 1. Eggbeater-Dandelion Design Configuration}

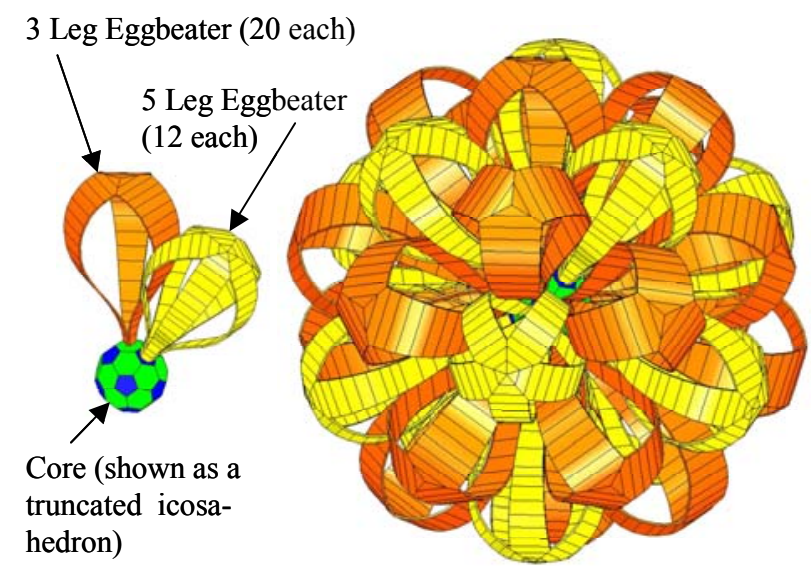

\section{Modeling Assumptions}

The Eggbeater-Dandelion concept has certain design characteristics that greatly influence dynamic modeling assumptions. Each of the 32 legs is designed to be flexible in the radial direction while stiff laterally. Radial flexibility allows the rover to absorb the impact forces associated with tumbling on the surface, while lateral stiffness avoids leg deformations that may result in leg entanglement. These characteristics will need to be modeled adequately in the simulation in order to capture the interaction of the legs with the ground, and the subsequent effects of the ground forces on the overall motion of the Tumbleweed.

Initial simulation attempts considered using a small point mass in place of each tumbleweed leg tip connected to the core by a spring-damper (Figure 2). Position and velocity of the point mass representing the tip is used to calculate interaction forces with the ground. This method is likely to produce reasonable results with two significant drawbacks. Modeling each leg with a small mass adds three degrees of freedom to the system for each leg, resulting in 96 additional degrees of freedom for a $32 \mathrm{leg}$ Tumbleweed. Small masses representing the legs would also produce dynamics in different time scales than the core body. In order to keep the simulation numerically stable, a small integration time step would have to be used, and combined with total degrees of freedom, the CPU requirements are likely to be great. A solution to the above problem is to use the singular perturbation theory. As the masses of the tumbleweed legs are reduced, the dynamic solution converges to a limiting case. The singularly perturbed solution is the fully converged solution as the masses of the leg tips approach zero. The singularly perturbed solution eliminates the equations of motion associated with the tumbleweed leg masses. At each integration time step, the solution for the locations of the leg tips in contact is computed. The locations of the tumbleweed leg tips are constrained by the ground and by the stiffness of the legs in the radial direction. 


\section{Figure 2. Modeling the Legs With Point Masses}

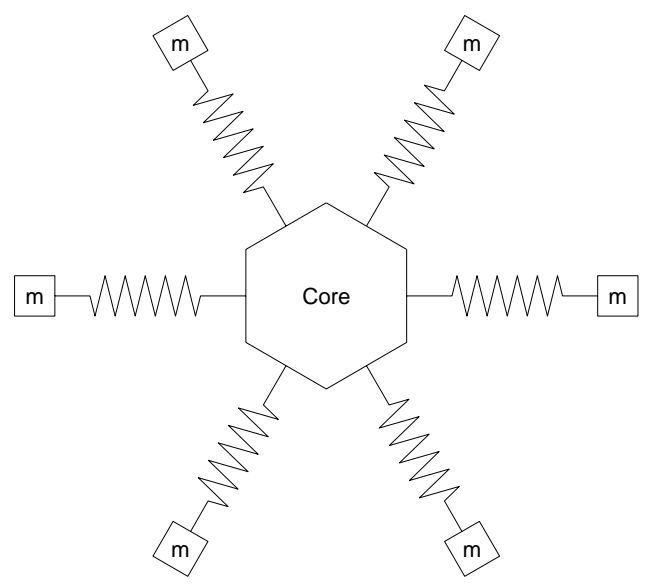

The flexibility of the legs is modeled with minimal numerical overhead. The simulation has six degrees of freedom, and the interaction of the legs and the ground is modeled as external forces on the rover. The ground is modeled as a constraint, and produces a force on the rover when any of the legs violate the constraint. Each leg is modeled as a spring-damper in the radial direction, and infinitely stiff laterally. The lateral constraint produces a force, and is accounted for in the simulation. The spring force is proportional to the amount of compression in the legs, and the damping force is a function of the compression rate. The ground is assumed to produce a force normal to the plane. The friction force is a function of the magnitude of the velocity vector of the leg projected on the surface, and the magnitude of the normal ground reaction force. The above assumptions produce dynamics that are reasonable and realistic. The simulation was verified by comparing the results to a Tumbleweed drop/roll test.

\section{Mathematical Description of the Ground Force}

Position, velocity, orientation, and rate of change of orientation of the Tumbleweed rover define the state vector. The orientation of the Tumbleweed is defined as the euler angles which transform the body axes to the inertial ground frame. The Tumbleweed is mostly symmetric about all axes and the body coordinate frame is chosen to be convenient. The inertial ground frame is defined with the North, East, and Down directions defining the $\mathrm{x}, \mathrm{y}$, and $\mathrm{z}$ axes respectively. The forces and moments are computed in terms of the state vector. Knowledge of the mass and rotational inertia of the Tumbleweed rover combined with applied forces are used to generate equations of motion. The orientation of each leg is known in the body frame of the Tumbleweed from its basic geometry. In the simulation, it is assumed that the orientation of the legs does not change when contacting the ground, i.e., the legs are infinitely stiff laterally. Below is the mathematical description of how the positions of velocities of leg tips in contact with the ground are computed. Figure 3 is a visual for the equations that follow. 


\section{Figure 3}

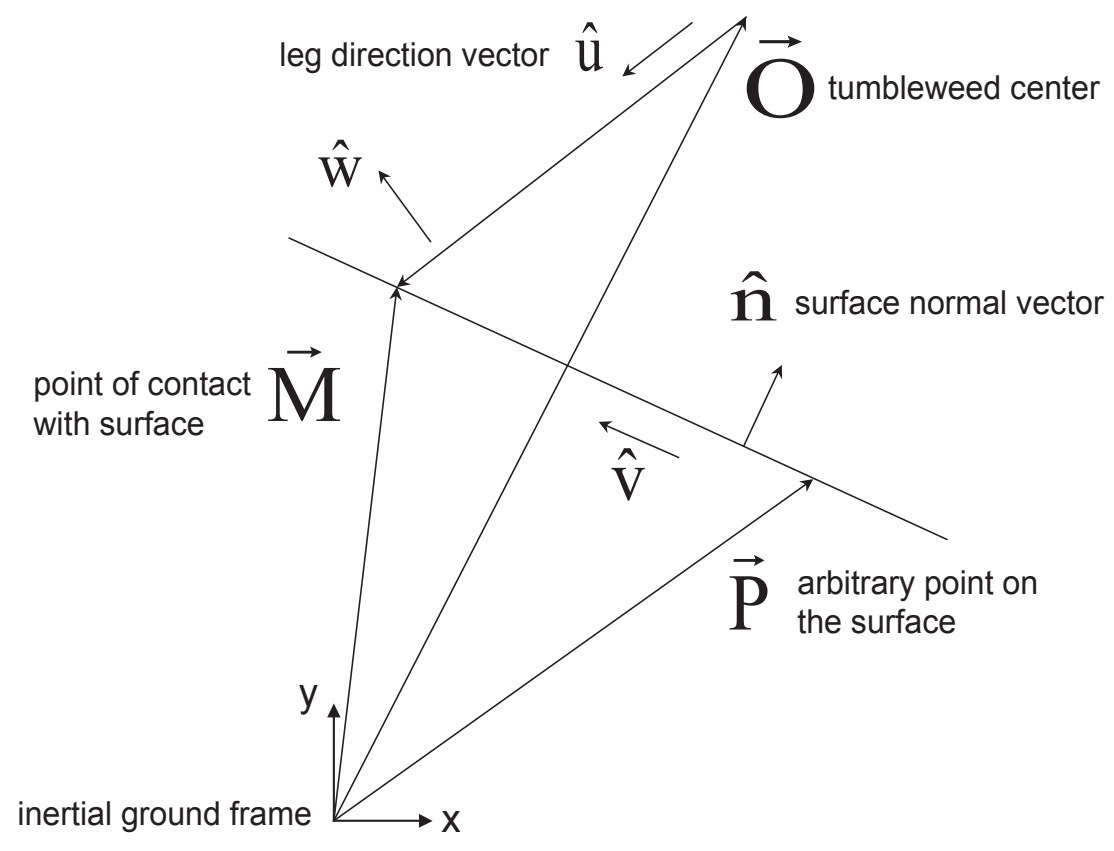

We must first find an expression for length of the leg in contact with the ground in terms of available parameters.

$$
\begin{array}{ll}
F_{K}=K\left(L-L_{0}\right) & \text { Leg compression due to stiffness } \\
L \hat{u}=\vec{M}-\vec{O} & \text { Definition of the where the tip of the tumbleweed leg is located } \\
\hat{n} \cdot(\vec{M}-\vec{P})=0 & \text { Dot product of perpendicular vectors equals zero } \\
\hat{n} \cdot(L \hat{u}+\vec{O}-\vec{P})=0 & \text { Substitution for M from equation (1) } \\
L \hat{n} \cdot \hat{u}+\hat{n} \cdot \vec{O}-\hat{n} \cdot \vec{P}=0 & \text { distributive law of dot product } \\
L=\frac{\hat{n} \cdot \vec{P}-\hat{n} \cdot \vec{O}}{\hat{n} \cdot \hat{u}} & \text { Solving for L }
\end{array}
$$

The leg compression rate needs to be calculated in order to compute the force due to damping. The leg compression rate is simply the time derivate of equation 6 . In this equation, $\hat{n}$ and $\overrightarrow{\mathrm{P}}$ are constants, while $\hat{\mathrm{u}}$ and $\overrightarrow{\mathrm{O}}$ are time dependent. 


$$
\begin{aligned}
& \dot{L}=\frac{d}{d t}\left(\frac{\hat{n} \cdot \vec{P}-\hat{n} \cdot \vec{O}}{\hat{n} \cdot \hat{u}}\right) \\
& \dot{L}=\frac{-(\hat{n} \cdot \dot{\vec{O}})(\hat{n} \cdot \hat{u})-(\hat{n} \cdot \dot{\hat{u}})(\hat{n} \cdot \vec{P}-\hat{n} \cdot \vec{O})}{(\hat{n} \cdot \hat{u})^{2}} \\
& \dot{L}=\frac{-(\hat{n} \cdot \dot{\vec{O}})(\hat{n} \cdot \hat{u})-(\hat{n} \cdot \dot{\hat{u}})(\hat{n} \cdot \vec{P})+(\hat{n} \cdot \dot{\hat{u}})(\hat{n} \cdot \vec{O})}{(\hat{n} \cdot \hat{u})^{2}} \\
& F_{C}=C \dot{L} \quad \operatorname{Leg} \operatorname{compression~due~to~stiffness~} \\
& F_{T}=F_{K}+F_{C} \quad \operatorname{Total} \text { force on each leg in contact } \\
& F_{T}=K\left(\frac{\hat{n} \cdot \vec{P}-\hat{n} \cdot \vec{O}}{\hat{n} \cdot \hat{u}}-L_{0}\right)+C\left(\frac{-(\hat{n} \cdot \dot{\vec{O}})(\hat{n} \cdot \hat{u})-(\hat{n} \cdot \dot{\hat{u}})(\hat{n} \cdot \vec{P})+(\hat{n} \cdot \dot{\hat{u}})(\hat{n} \cdot \vec{O})}{(\hat{n} \cdot \hat{u})^{2}}\right)
\end{aligned}
$$

The normal ground reaction force and the friction force are the only external forces acting on each leg in contact. The ground force is assumed to act in the direction normal to the surface, and the friction force direction is opposite to the projected velocity of the tip. Because the legs are constrained in the lateral direction, there is a constraint force normal to the direction of the leg (Figure 3). A good way to visualize this constraint force is to think of each leg as a massless, frictionless ball in a tube, with the ball representing the Tumbleweed leg tip (Figure 4). The constraint force is the force of the cylinder wall on the ball, in the direction normal to the tube. The normal ground reaction force, the friction force, the total spring force, and the lateral constraint force must add to zero. We know that the total spring force and the lateral constraint force are perpendicular to each other; therefore, their dot product is equal to zero. This property is used to eliminate the constraint force from the equations.

The friction force is calculated by multiplying the projected velocity of the Tumbleweed leg in contact, the normal ground reaction force, and the dynamic friction coefficient.

$$
\begin{array}{ll}
V_{F} \hat{v}=-(\vec{V}-(\vec{V} \cdot \hat{n}) \hat{n}) & \text { Subtracting the normal component of velocity } \\
F_{F}=\mu F_{N} V_{F} & \text { Friction force } \\
\hat{u} \cdot \hat{w}=0 & \text { Perpendicular vectors } \\
F_{N} \hat{n}+F_{T} \hat{u}+F_{W} \hat{w}+F_{F} \hat{v}=0 & \text { Sum of all forces at the tip } \\
F_{N} \hat{n} \cdot \hat{u}+F_{T} \hat{u} \cdot \hat{u}+F_{W} \hat{w} \cdot \hat{u}+F_{F} \hat{v} \cdot \hat{u}=0 & \text { Dot all terms by the u vector } \\
F_{N} \hat{n} \cdot \hat{u}+F_{T}+F_{F} \hat{v} \cdot \hat{u}=0 & \text { Substitute for friction force } \\
F_{N} \hat{n} \cdot \hat{u}+F_{T}+\mu F_{N} V_{F} \hat{v} \cdot \hat{u}=0 &
\end{array}
$$




$$
F_{N}=-\frac{F_{T}}{\left(\hat{n} \cdot \hat{u}+\mu F_{N} V_{F} \hat{v} \cdot \hat{u}\right)}
$$

The normal force and the friction force are applied to the Tumbleweed for each leg in contact with the ground as external forces.

Figure 4. Ball in a Tube Analogy

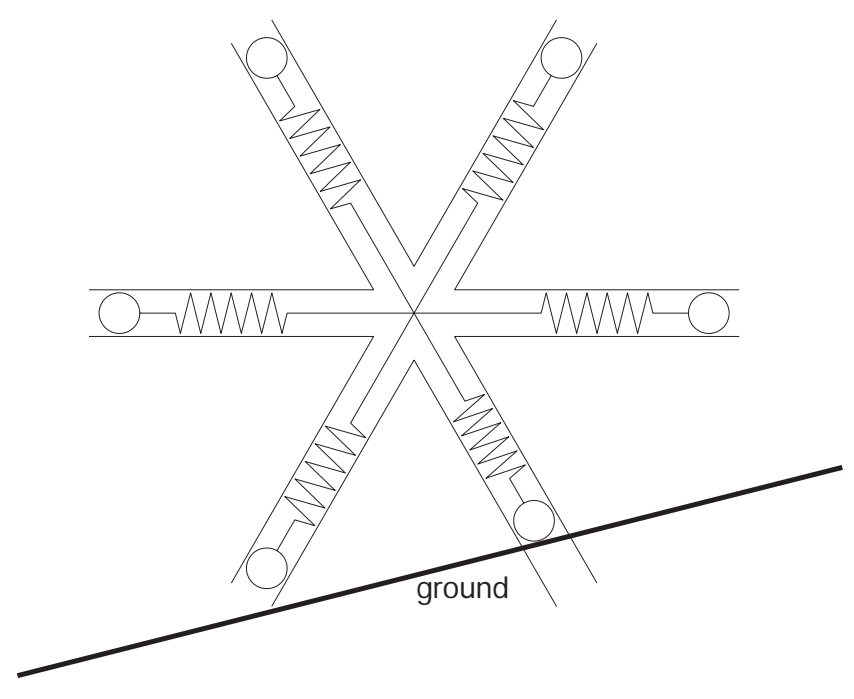

\section{Dynamic Model Verification}

A scaled down test article of the Eggbeater-Dandelion concept was built and tested in a dynamic testing facility at NASA Langley Research Center (Figure 5). These tests were used to characterize the tumbling and rolling dynamics, particularly in the presence of structural nonlinearity. ${ }^{2}$ Videogrammetry was used to determine the motion of targets, each of which were mounted to the article's outer structure at the end of each leg. An array of five cameras was positioned to provide tracking of at least three targets in multiple camera views as the article was dropped and rolled down an inclined ramp. This allowed resolution of each visible target's 3dimensional location in a room-fixed reference frame during each test run. Processing position data from the tracked targets produced an estimate of the center of mass position along the test trajectory. The processed center of mass data from a set of these test runs was compared with results of the Matlab simulation based on the dynamic equations developed in this paper. Model input parameters not explicitly measured apriori, specifically leg damping and friction, were tuned to match the test data. This simulation tool was able to successfully predict the dynamic motion of tumbleweed during bounce tests (Figure 6). For a complete description of the test setup and a detailed discussion of the test results and simulation comparisons see Reference 2. This reference also shows results of simulations used to demonstrate wind-blown mobility of the full-scale Mars Tumbleweed from a resting condition on flat terrain using a representative set of 
Eggbeater-Dandelion design and Mars environmental parameters. These results demonstrated good agreement with basic feasibility studies described in Reference 1.

\section{Conclusions}

This paper describes the development of a math model for simulation of tumbling dynamics of a candidate Tumbleweed rover, known as the Eggbeater-Dandelion. The model was successfully validated in dynamics testing of a scaled test article and was used to confirm windblown mobility in representative Mars conditions for the full-scale vehicle. Future dynamics studies of this Tumbleweed concept will include studies of wind-blown mobility along simulated Mars terrain at selected sites, and studies of controlled mobility using actuation of the Eggbeater legs including start/stop, downwind, and crosswind motion.

Figure 5. Eggbeater-Dandelion Dynamic Test Setup

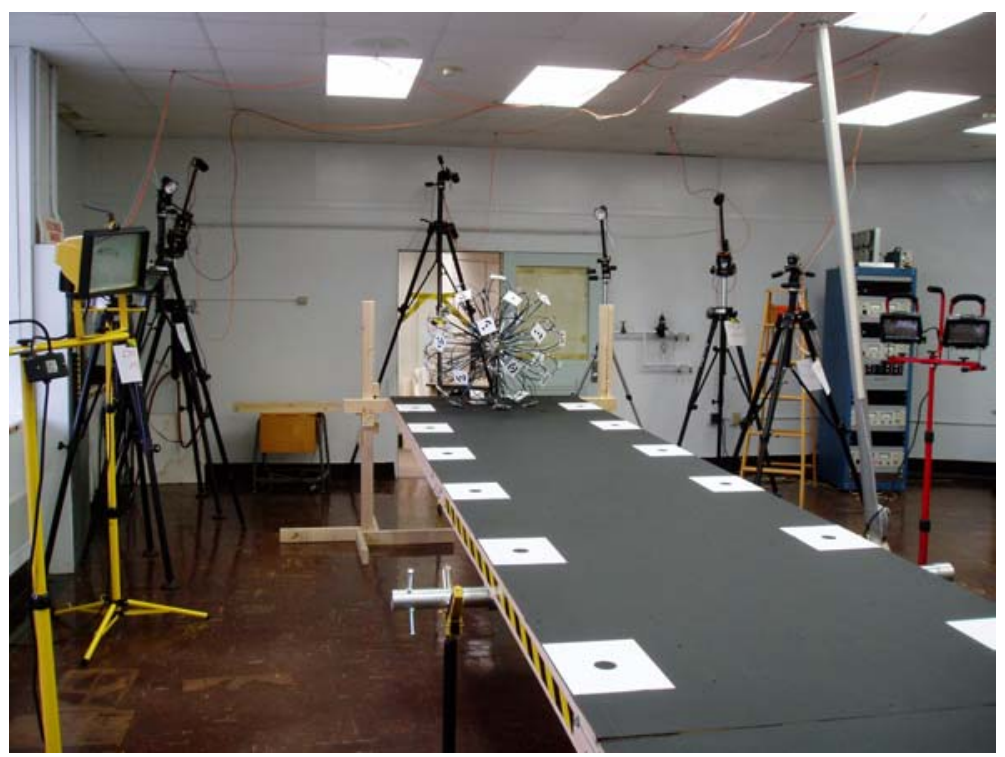




\section{Figure 6. Simulation Comparison to a Dynamic Test run}

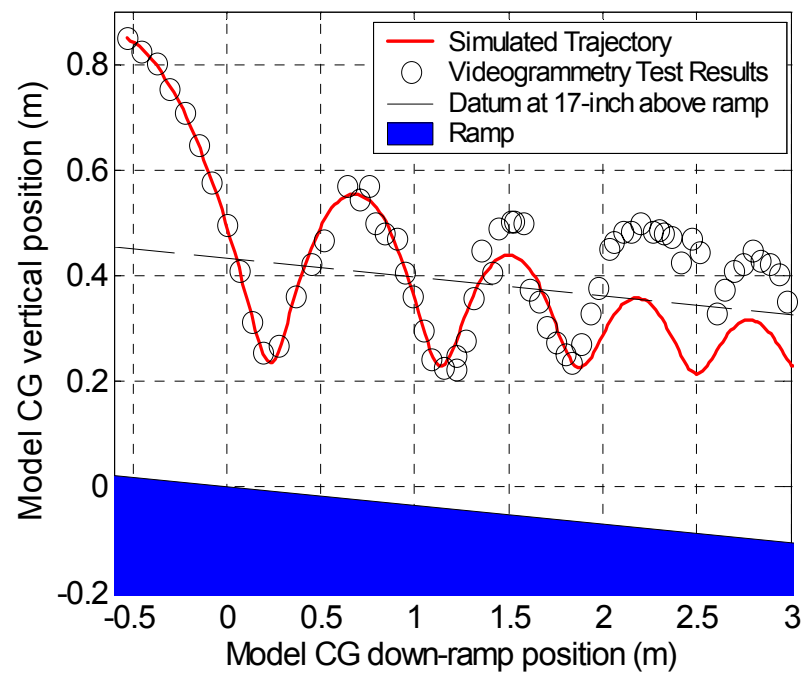

\section{Acknowledgements}

The authors would like to acknowledge Jeff Antol and Greg Hajos for leading the project. Steve Harris was instrumental in design of the Eggbeater-Dandelion concept. He also provided the CAD drawing in this paper. Hans Seywald of Analytical Mechanics Associates is recognized for sharing his knowledge about the singular perturbation theory.

\section{Reference}

1. Antol, J., Calhoun, P., Flick, J., Hajos, G., Kolacinski, R., Minton, D., Owens, R., Parker, J., "Low Cost Mars Surface Exploration: The Mars Tumbleweed," NASA TM-2003212411, Aug. 2003.

2. Calhoun, P., Harris, S., Raiszadeh, B., Saleski, K., "Conceptual Design and Dynamics Testing and Modeling of a Mars Tumbleweed Rover," AIAA Paper 2005-0247, 2005. 